\title{
Interactions of free-living amoebae with the rice fungal pathogen, Rhizoctonia solani
}

\author{
John J. Long ${ }^{1,2}$, Emily K. Luna ${ }^{2}$, Mary Jackson ${ }^{3}$, William Wheat ${ }^{3}$, Courtney E. Jahn ${ }^{2}$ and Jan E. Leach ${ }^{2 *}$ (i)
}

\begin{abstract}
Objective: Rhizoctonia solani is a soil-borne fungal pathogen of many important crop plants. In rice, R. solani causes sheath blight disease, which results in devastating grain yield and quality losses. Few methods are available to control this pathogen and classic single gene resistance mechanisms in rice plants have not been identified. We hypothesize that alternate means of control are available in the environment including free-living amoebae. Amoebae are soil-, water- and air-borne microorganisms that are predominantly heterotrophic. Many amoeba species are mycophagous, and several harm their prey using mechanisms other than phagocytosis. Here, we used light and scanning electron microscopy to survey the interactions of $R$. solani with four amoeba species, with the goal of identifying amoebae species with potential for biocontrol.

Results: We observed a wide range of responses during interactions of $R$. solani with four different free-living amoebae. Two Acanthamoeba species encyst in co-cultures with $R$. solani at higher rates than medium without $R$. solani. Vermamoeb a vermiformis (formerly Hartmanella vermiformis) attach to $R$. solani mycelium and are associated with mycelial shriveling and perforations of fungal cell walls, indicating an antagonistic interaction. No phenotypic changes were observed in co-cultures of Dictyostelium discoideum and R. solani.
\end{abstract}

Keywords: Free-living amoebae, Rhizoctonia solani, Sheath blight, Acanthamoeba, Dictyostelium, Vermamoeba

\section{Introduction}

Rhizoctonia solani is a significant fungal pathogen of rice that is particularly difficult to control. This fungus causes rice sheath blight, a necrotic disease characterized by lesions initiating at the apical ends of rice sheaths. As a persistent and virulent soil-borne pathogen, $R$. solani can reduce yields by half in conducive environmental conditions [1]. The fungus survives in soil and in fields by forming sclerotia, which are condensed bodies of fungal hypha that may survive in soil for up to 2 years $[1,2]$. The broad host spectrum of $R$. solani allows the fungus to infect alternative hosts as another means to remain in an environment [3]. Compounding the issue of pathogen persistence is that there are currently no plant

\footnotetext{
*Correspondence: Jan.Leach@colostate.edu

2 Department of Bioagricultural Sciences and Pest Management, Colorado State University, Fort Collins, CO, USA

Full list of author information is available at the end of the article
}

disease resistance genes identified for control of R. solani, although potential quantitative trait loci that incrementally increase plant resistance have been identified $[1,4$, 5]. To reduce instances and severity of outbreaks from $R$. solani, additional methods of control are needed. To that end, we explored interactions of the fungus with freeliving amoebae, with a view towards adapting amoebae antagonistic to $R$. solani as biological control agents.

Historical studies of the interactions of free-living amoebae and $R$. solani are limited. In one study, sclerotia and hyphae inoculated into soil samples showed signs of extensive predation by mycophagous protozoa [6]. Amoebae recovered from the soil were identified to be a species of Thecamoeba based on morphology.

Interactions among other fungi and amoebae have been well described. After incubation in soil, conidia of Cochliobolus sativus were lysed, with multiple perforations visible in the cell wall, suggestive of amoebal predation [7]. 
Some amoebae engulf entire conidia, after which they encyst, slowly digesting their prey inside the cyst $[7,8]$. A member of the genus Acanthamoeba also preys on a variety of fungi pathogenic to mammals, such as Blastomyces dermatitidis and Cryptococcus neoformans [9]. Interestingly, some strains of $C$. neoformans survive inside $A$. castellanii, and use the amoebae as a reservoir for future infections [9].

Given their proximity in the phytobiome, we hypothesize that free-living amoebae interact with $R$. solani. To study these interactions, we observed different amoebal species (A. castellanii, A. polyphaga, D. discoideum and $V$. vermiformis) after co-culture with $R$. solani by light and fluorescence microscopy. Of the four amoebae tested, we found that only $V$. vermiformis caused detrimental changes in the fungal hyphae, and we further explored these interactions with scanning electron microscopy (SEM).

\section{Main text \\ Methods}

\section{Amoebae and fungi culturing conditions}

Acanthamoeba species were cultured at $28{ }^{\circ} \mathrm{C}$ in a modified peptone, yeast and glucose medium (PYG), $V$. vermiformis was cultured at $28^{\circ} \mathrm{C}$ in a modified peptone, yeast extract, liver digest, hemin and serum medium (PYNFH) and $D$. discoideum was maintained at room temperature in a modified rich axenic medium (HL5) [10]. Amoebae cultures were inoculated from frozen stocks into $100 \times 15 \mathrm{~mm}$ petri dishes with $30 \mathrm{~mm}$ walls holding $10 \mathrm{~mL}$ of medium supplemented with Gibco penicillin/ streptomycin (Invitrogen; California, United States) to a $1 \times$ working concentration. Once initial cultures reached turbidity, Acanthamoeba species and $V$. vermiformis were passaged every 5 days by transferring $500 \mu \mathrm{L}$ of culture into $10 \mathrm{~mL}$ of fresh medium. $D$. discoideum was passaged every 3 days. Amoebae cultures were passaged no more than three times prior to use in our studies.

Rhizoctonia solani was cultured on $1 / 2$ strength potato dextrose agar (PDA; Difco) from frozen stocks prepared on barley seeds according to [11]. Initial cultures were incubated at $22{ }^{\circ} \mathrm{C}$ with $16 \mathrm{~h}$ of light for 10 days, then stored at $4{ }^{\circ} \mathrm{C}$ for use as a source of agar plugs. Source plates were kept for up to 3 weeks before starting new cultures from stock. Agar plugs $7.5 \mathrm{~mm}$ in diameter were subcultured onto autoclaved cellophane overlaid onto $1 / 2$ strength PDA and incubated for 7-10 days at the above conditions before use in experiments.

\section{Co-cultures of amoebae and R. solani}

Confluent cultures of amoeba were starved overnight in diluted medium at the temperatures described above, except for $D$. discoideum, which was kept in full strength medium. Acanthamoeba were starved in $1 / 5$ strength PYG while $V$. vermiformis were starved in $1 / 2$ strength PYNFH; media were diluted using Page's modified amoeba saline (PAS) [10]. Amoebal cell density was calculated using a direct cell counting method involving trypan blue exclusion and a hemocytometer. Only cultures with over $90 \%$ viable trophozoites were used. Amoebae cultures were adjusted to concentrations of $2 \times 10^{5}$ cells $/ \mathrm{mL}$ in fresh, diluted medium.

Plugs of fungal mycelia were cut with a sterilized borer with an internal diameter of $5 \mathrm{~mm}$. Fungal plugs were removed from the agar plate using sterile forceps and rinsed once in sterile, distilled water and transferred to a $1.5 \mathrm{~mL}$ centrifuge tube. $500 \mu \mathrm{L}$ of amoebae culture was added to each tube containing fungi. Each amoebae and fungal combination were prepared in triplicate for sampling at each time point of 0, 24, and $48 \mathrm{~h}$. Co-cultures with Acanthamoeba sp. or $V$. vermiformis were incubated at $28^{\circ} \mathrm{C}$ and cultures with $D$. discoideum were incubated at $22^{\circ} \mathrm{C}$.

\section{Microscopy}

Co-cultures were centrifuged at $150 \times \mathrm{G}$ for $3 \mathrm{~min}$ and the supernatant was removed. Pellets were washed three times with $500 \mu \mathrm{L}$ of PAS by centrifugation at $150 \times \mathrm{G}$ for 3 min each time. After washing, pellets were fixed with $100 \mu \mathrm{L}$ of $4 \%$ paraformaldehyde for $48 \mathrm{~h}$. After fixation, samples were pelleted and then suspended in $30 \mu \mathrm{L}$ of PAS. For viability staining, samples were first dyed with $4 \mu \mathrm{L}$ of $8 \mathrm{mg} / \mathrm{mL}$ fluorescein diacetate (FDA) and 25 $\mu \mathrm{L}$ of $2 \mathrm{mg} / \mathrm{mL}$ propidium iodide (PI) for $15-20 \mathrm{~min}$ in the dark. Samples were then washed and fixed as noted above, then mounted on glass microscope slides.

Standard light and fluorescence microscopy were conducted on a Zeiss Axioskop microscope fitted with Chroma Technology filters. FDA was visualized using 480 and $535 \mathrm{~nm}$ excitation and emission filters, respectively, and PI was visualized with $535 \mathrm{~nm}$ excitation and $610 \mathrm{~nm}$ emission filters. Images were captured and falsecolored using the Prog Res Capture Pro software (Jenoptik) and multi-color images were obtained by overlaying images from the FDA and PI channels. Adobe Photoshop CS6 was used to crop and adjust images.

Confocal laser scanning microscopy was carried out on a Zeiss LSM 510 inverted microscope. Samples were excited with a $488 \mathrm{~nm}$ laser and emission filters were set to 480 and $590 \mathrm{~nm}$ for FDA and PI, respectively. At $400 \times$ and $630 \times$ magnification, three random fields were taken per sample and images were taken at ten different depths in $0.5-1.5 \mu \mathrm{m}$ increments. Images were merged into one using the Zeiss Zen 2009 software.

\section{Scanning electron microscopy}

Co-cultures were prepared such that (1) the two organisms could directly contact one another or (2) the two 
species were prevented from physical contact. In the former, amoebae were adjusted to a concentration of $2 \times 10^{5}$ trophozoites $/ \mathrm{mL}$ and $10 \mathrm{~mL}$ of the culture was added to a high-walled petri dish. Fifteen plugs of $R$. solani were added to the culture and the dish was sealed with Parafilm then stored in a plastic bag. To separate the two species, co-cultures of $V$. vermiformis and $R$. solani for SEM were prepared following a modified procedure from Homma and Ishii [6]. Two nucleopore membranes $(25 \mathrm{~mm}$ in diameter; Whatman \#110610; Maidstone, United Kingdom) with $1.0 \mu \mathrm{m}$ pores were used to sandwich three fungal discs, the edges of the membrane sandwiches were sealed with silicon vacuum grease. Five sandwiched membranes were added to a petri dish containing $2 \times 10^{6} \mathrm{~V}$. vermiformis trophozoites at $10 \mathrm{~mL}$ of final volume. Co-cultures were incubated at $22^{\circ} \mathrm{C}$ with $16 \mathrm{~h}$ of light. At $0,2,6,12$, and $24 \mathrm{~h}$, three disks from each culture were transferred to individual micro-centrifuge tubes and centrifuged once at $150 \times \mathrm{G}$ for $3 \mathrm{~min}$. Supernatant was discarded and samples were washed once in $500 \mu \mathrm{L}$ PAS. After centrifugation and removal of the wash, samples were fixed in $2.5 \%$ glutaraldehyde buffered in $0.15 \mathrm{M}$ Sorensen's phosphate buffer, $\mathrm{pH} 7.0\left(22^{\circ} \mathrm{C}\right.$ for $30-60 \mathrm{~min}$, followed by $4{ }^{\circ} \mathrm{C}$ ). Tissue samples were dehydrated through a graded ethanol series, followed by final dehydration using a BioRad E3000 critical point dryer (Quorum Technologies, East Sussex, England). All samples prepared for SEM were sputter coated with $10 \mathrm{~nm}$ gold, imaged at $5 \mathrm{kV}$ with a JEOL JSM6500F Field Emission Scanning Electron Microscope. All images were captured as tiff files.

\section{Results with discussion \\ Microscopy reveals a diverse array of reactions}

Different genera of amoebae trophozoites interacted differently with $R$. solani mycelium in co-cultures. At $48 \mathrm{~h}$, the two Acanthamoeba species encysted at higher rates than the amoebae-only control in PAS non-nutrient media (Fig. 1a-c). The cysts clumped together around the mycelium rather than floating free in the culture, an observation noted in co-cultures prepared with and without centrifugation. No changes in fungal mycelium
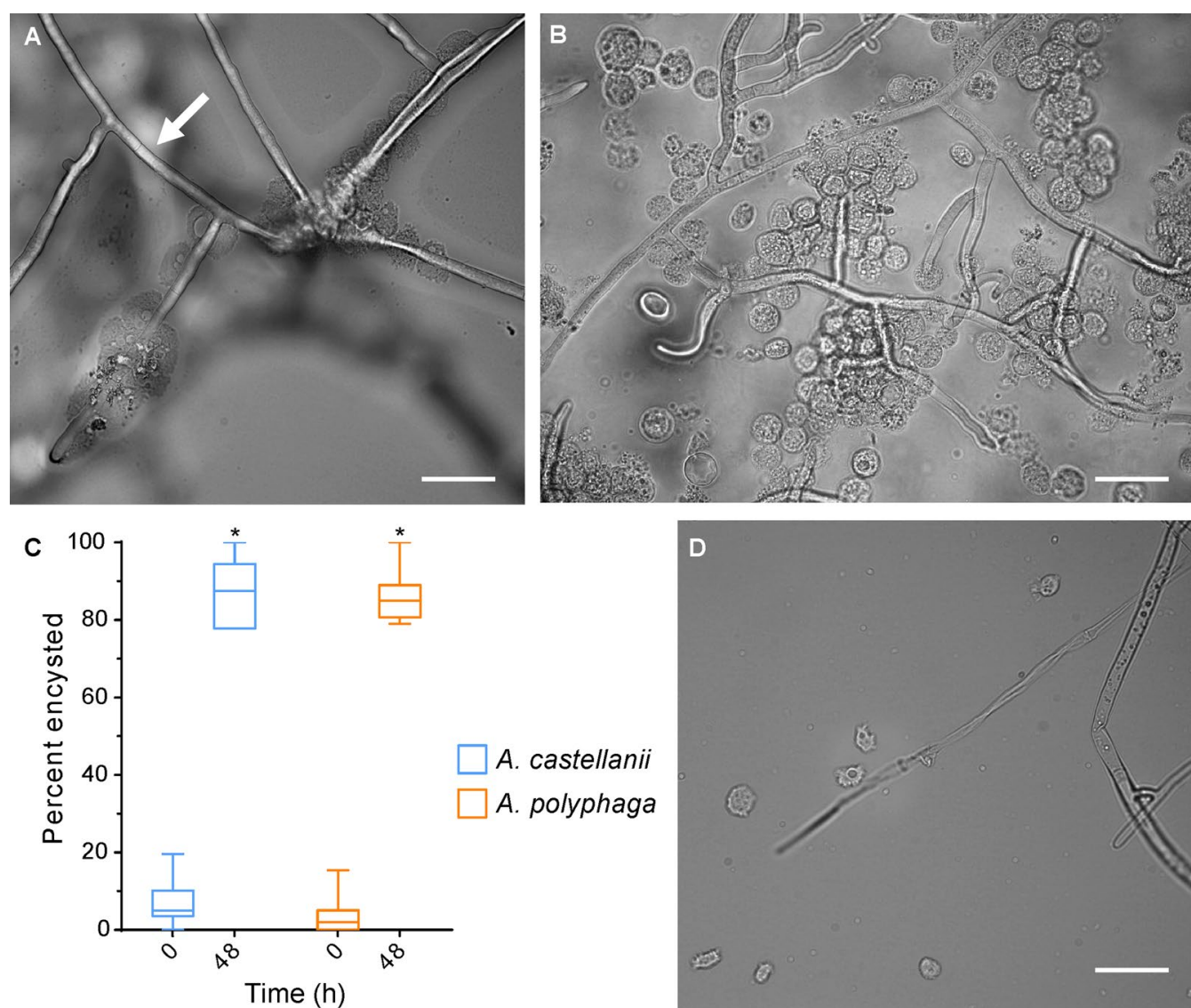

A. castellanii

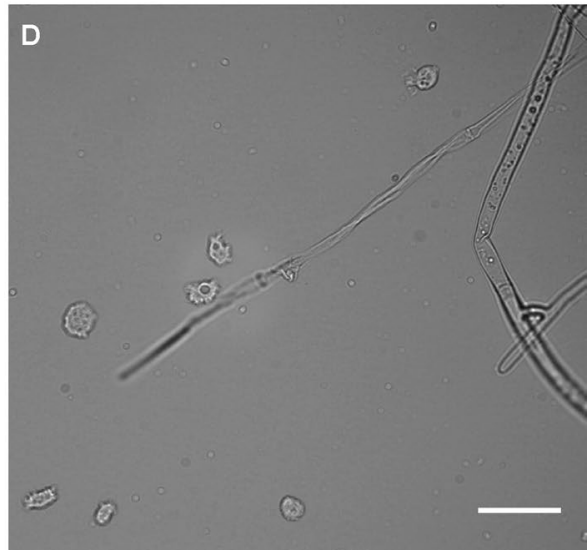

Fig. 1 Light microscopy of amoeba and R. solani co-cultures. a A. castellanii and R. solani after 48 h. b A. polyphaga and R. solani after 24 h. The smooth and straight cell walls of $R$. solani are easily visible, indicated by a white arrow. c Encystment of A. castellanii and A. polyphaga after co-culture with $R$. solani, stars denote $p<0.0001$ calculated by a Student's $T$ test. $\mathbf{d} D$. discoideum and $R$. solani after $24 \mathrm{~h}$. All images were taken at $\times 630$ magnification and scale bars are $10 \mu \mathrm{m}$ 
were observed after co-cultivation. The hyphae remained intact with no visible perforations. Nuclei, stained red with propidium iodide, were not disrupted. In addition, the hyphal cell wall remained smooth and mycelia were branched at right angles, as is typical for $R$. solani.

Dictyostelium discoideum did not have any apparent physical interaction with the fungal hyphae. Under light microscopy, the amoebae did not attach to hyphae or form sporulating bodies, a sign of nutrient deprivation or environmental incompatibility. The fungal hyphae were not visibly altered, and mycelium remained intact and with no observed perforations (Fig. 1d).

Of the four amoebae tested, only $V$. vermiformis had a noticeable effect on the fungus. Trophozoites were physically attached to the mycelium and remained viable for at least the $24 \mathrm{~h}$ of co-culture (Fig. 2). Scanning

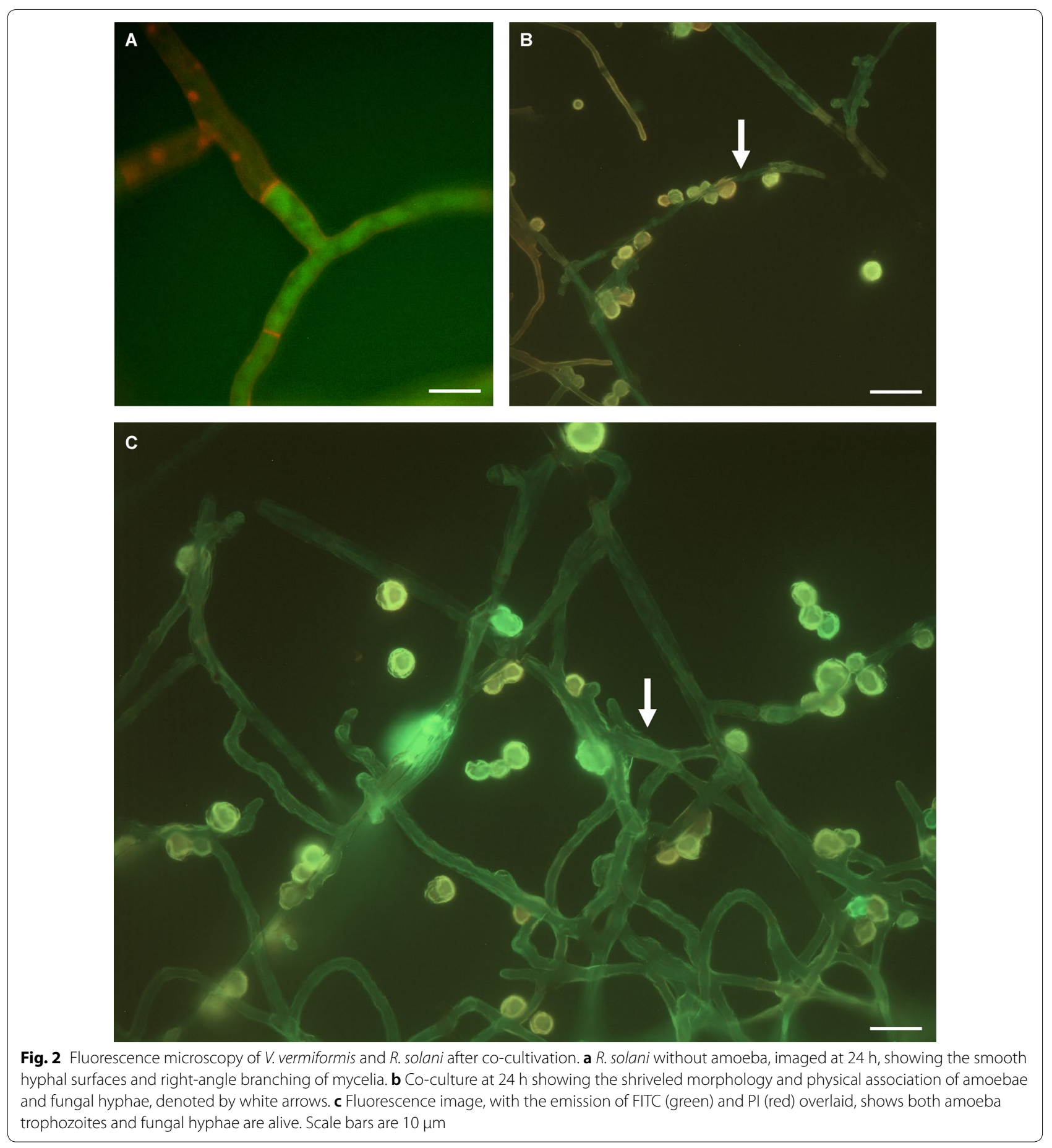


electron microscopy revealed that, after $24 \mathrm{~h}$ co-cultivation, the surface of $R$. solani mycelia became mottled and shriveled (Fig. $3 \mathrm{a}-\mathrm{c}$ ). The shriveling was not observed when the fungus was cultured in PYNFH medium without $V$. vermiformis (Fig. 3d). The hyphal appearance after co-cultivation with $V$. vermiformis was in stark contrast from the smooth cell walls and branching hyphae of normal $R$. solani [12]. Interestingly, the shriveled appearance of hyphae occurred even when $V$. vermiformis and $R$. solani were physically separated by membranes (Fig. 3e-g). While transient contact can occur through the $1 \mu \mathrm{m}$ pores of the membrane, the amoebae cannot physically wrap around the mycelium. Perforations with smooth and rounded edges, although rare, were detected on the fungal mycelium of the physically separated co-cultures.

It is not surprising that the panel of amoebae presented a variety of interactions with the fungi. Some bacterial species are known to antagonize amoebae and force encystment or even lyse the amoebae [13-15]. The cause
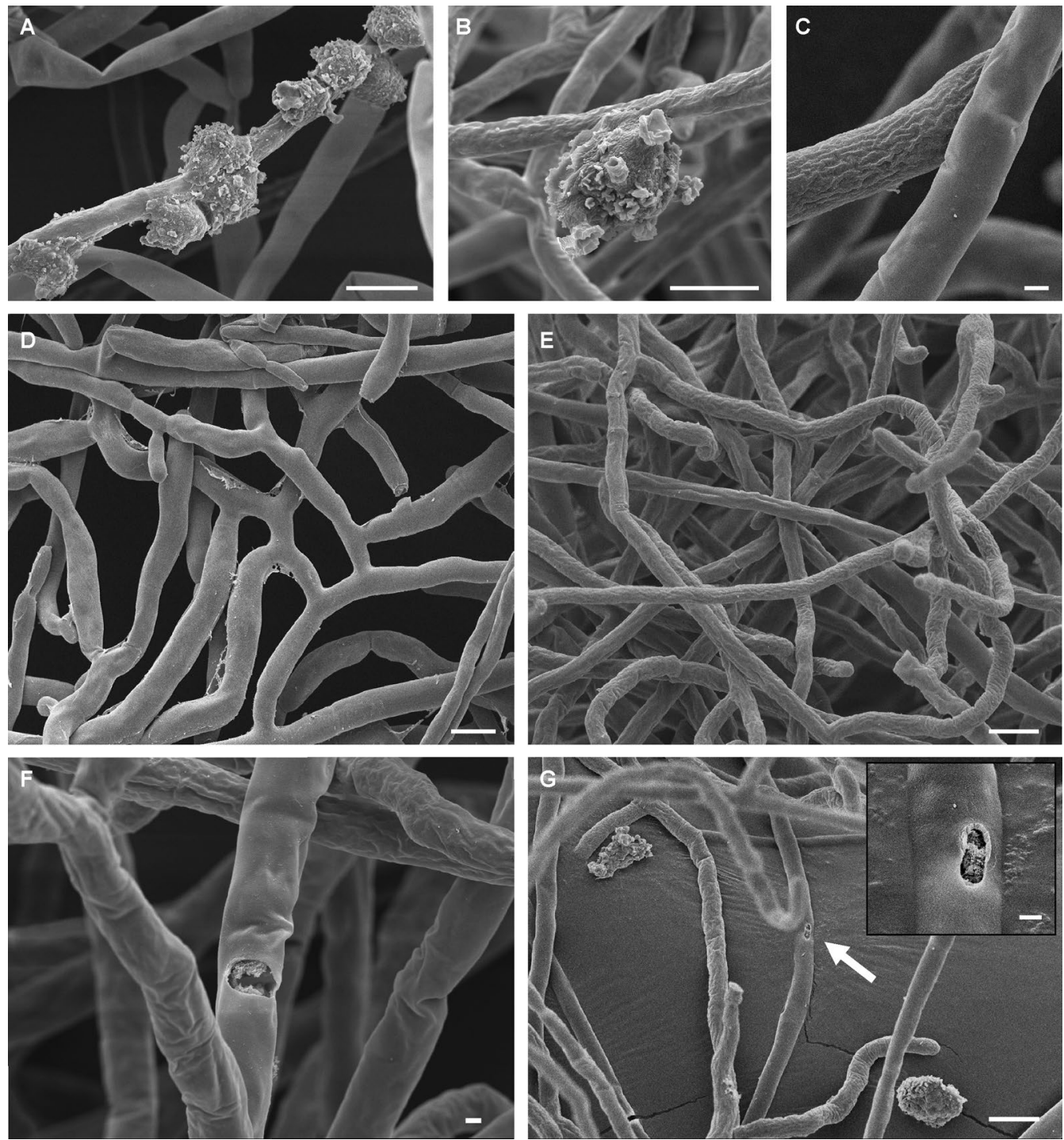

Fig. 3 Scanning electron microscopy of V. vermiformis and R. solani after co-culture. Organisms were not separated by a nucleopore membrane; $24 \mathrm{~h}$ co-culture. a Micrographs show $V$. vermiformis trophozoites partially wrapped around $R$. solani hyphae. $\mathbf{b}$ A close-up image of a trophozoite and shriveled hypha. c A close-up micrograph of hyphae at varying intensities of shriveling. Organisms were separated by a nucleopore (1 $\mu \mathrm{m})$ membrane and evaluated at 0 (d) and $24 \mathrm{~h} \mathrm{(e)} \mathrm{after} \mathrm{co-cultivation.} \mathrm{The} \mathrm{hyphae} \mathrm{of} R$. solani develop a shriveled exterior even when separated from the amoeba after $24 \mathrm{~h}$. $\mathbf{f}, \mathbf{g}$ A perforation with smooth edges detected on the hyphae after co-culturing with amoebae in the same medium. Scale bars in $\mathbf{a}, \mathbf{b}, \mathbf{d}, \mathbf{e}$ and $\mathbf{g}$ are $10 \mu \mathrm{m}$; scale bars in $\mathbf{c}, \mathbf{f}$ and inset of $\mathbf{g}$ are $1 \mu \mathrm{m}$ 
of encystment in Acanthamoeba is not known at this time, and we have not ruled out nutrient deprivation as the factor. To our knowledge, there are no characterized mechanisms of anti-amoebal activity from $R$. solani. The reason for and the nature of the Acanthamoeba cysts' physical association with the fungal hyphae is not understood. One explanation could be that Acanthamoeba trophozoites attach and attempt to feed, and in doing so encounter something that stimulates encystment.

The shriveling response of the fungi after exposure to $V$. vermiformis suggests antagonism. Because the same response occurred with and without direct contact of the two organisms, it is possible the fungi is reacting to a secreted amoebal factor. The extent of the response is still uncharacterized and the effects of amoebal secretions on fungi are not well studied. However, some plant extracts are capable of causing similar responses in $R$. solani $[12$, 16]. In those cases, shriveling of $R$. solani after contact with plant extracts was caused by collapse of the cell wall. Lastly, the observations of perforations of hyphae, although rare, in the membrane-separated cultures suggest that $V$. vermiformis can puncture $R$. solani cell walls. In cultures not separated by a membrane, it is possible that perforations are obscured by an attached amoeba.

\section{Limitations}

1. The encystment of Acanthamoeba species early in interactions with $R$. solani may or may not have required fungal contact.

2. The presence of $V$. vermiformis trophozoites attached to mycelia in the SEM studies may have obscured observation of perforations in fungal cell walls.

\begin{abstract}
Abbreviations
SEM: scanning electron microscopy; PYG: modified peptone, yeast and glucose medium; PYNFH: modified peptone, yeast extract, liver digest, hemin and serum medium; HL5: modified rich axenic medium; PDA: potato dextrose medium; PAS: Page's modified amoeba saline; FDA: fluorescein diacetate; PI: propidium iodide.
\end{abstract}

\section{Acknowledgements}

We would like to thank Kim Vanderpool for her help in conducting scanning electron microscopy.

\section{Authors' contributions}

$J J L, E K L, M J, W W, C E J$, and JEL designed the experiments. JJL and EL performed the experiments. JJL, CEJ and JEL wrote the manuscript. All authors read and approved the final manuscript.

\section{Funding}

Research was sponsored by the U.S. Army Research Office and the Defense Advanced Research Projects Agency and was accomplished under cooperative Agreement Number W91 1NF-15-2-0124. The funding supported JJL, and the experiments performed herein. The views and conclusions contained in this document are those of the authors and should not be interpreted as representing the official policies, either expressed or implied, of the Army Research Office, DARPA, or the U.S. Government. The U.S. Government is authorized to reproduce and distribute reprints for Government purposes notwithstanding any copyright notation hereon.

\section{Availability of data and materials}

All data generated or analyzed during this study are included in this published article.

Ethics approval and consent to participate

Not applicable.

Consent for publication

Not applicable.

\section{Competing interests}

The authors declare that they have no competing interests.

\section{Author details}

${ }^{1}$ Department of Plant Pathology, The Ohio State University, Columbus, $\mathrm{OH}$, USA. ${ }^{2}$ Department of Bioagricultural Sciences and Pest Management, Colorado State University, Fort Collins, CO, USA. ${ }^{3}$ Mycobacteria Research Laboratories, Department of Microbiology, Immunology, and Pathology, Colorado State University, Fort Collins, CO, USA.

Received: 11 October 2019 Accepted: 12 November 2019

Published online: 15 November 2019

References

1. Lee FN. Rice sheath blight: a major rice disease. Plant Dis. 1983;67:829.

2. Ghosh S, Kanwar P, Jha G. Identification of candidate pathogenicity determinants of Rhizoctonia solani AG1-IA, which causes sheath blight disease in rice. Curr Genet. 2018. https://doi.org/10.1007/s0029 4-017-0791-7.

3. Anderson NA. The genetics and pathology of Rhizoctonia Solani. Annu Rev Phytopathol. 1982;20(1):329-47.

4. Zhao CJ, Wang AR, Shi YJ, Wang LQ, De Liu W, Wang ZH, et al. Identification of defense-related genes in rice responding to challenge by Rhizoctonia solani. Theor Appl Genet. 2008;116(4):501-16.

5. Channamallikarjuna V, Sonah H, Prasad M, Rao GJN, Chand S, Upreti HC, et al. Identification of major quantitative trait loci qSBR11-1 for sheath blight resistance in rice. Mol Breed. 2010. https://doi.org/10.1038/ nrg2270.

6. Homma Y, Ishii M. Perforation of hyphae and sclerotia of Rhizoctonia solani Kühn by mycophagous soil amoebae from vegetable field soils in Japan. Jap J Phytopathol. 1984;50(2):229-40.

7. Anderson TR, Patrick ZA. Soil Vampyrellid amoebae that cause small perforations in conidia of Cochliobolus sativus. Soil Biol Biochem. 1980;12(2):159-67.

8. Old KM. Perforation and lysis of fungal spores by soil amoebae. Ann Appl Biol. 1978;89:128-31.

9. Malliaris SD, Steenbergen JN, Casadevall A. Cryptococcus neoformans var. gattii can exploit Acanthamoeba castellanii for growth. Med Mycol. 2004;42(2):149-58.

10. Wheat $\mathrm{WH}$, Casali AL, Thomas $V$, Spencer JS, Lahiri R, Williams DL, et al. Long-term survival and virulence of Mycobacterium leprae in amoebal cysts. PLoS Negl Trop Dis. 2014;8(12):e3405.

11. Webb KM, Hill AL, Laufman J, Hanson LE, Panella L. Long-term preservation of a collection of Rhizoctonia solani using cryogenic storage. Ann Appl Biol. 2011;158(3):297-304.

12. Plodpai P, Chuenchitt S, Petcharat V, Chakthong S, Voravuthikunchai SP. Anti-Rhizoctonia solani activity by Desmos chinensis extracts and its mechanism of action. Crop Prot. 2013;43:65-71.

13. El-Etr SH, Margolis JJ, Monack D, Robison RA, Cohen M, Moore E, et al. Francisella tularensis Type A strains cause the rapid encystment of Acanthamoeba castellanii and survive in amoebal cysts for three weeks postinfection. Appl Environ Microbiol. 2009;75(23):7488-500. 
14. Greub G, Raoult D. Microorganisms resistant to free-living amoebae. Clin Microbiol Rev. 2004;17(2):413-33.

15. Long JJ, Jahn CE, Sánchez-Hidalgo A, Wheat W, Jackson M, GonzalezJuarrero M, et al. Interactions of free-living amoebae with rice bacterial pathogens Xanthomonas oryzae pathovars oryzae and oryzicola. PLoS ONE. 2018;13(8):e0202941.

16. Castillo F, Hernández D, Gallegos G, Mendez M, Rodríguez R, Reyes A, et al. In vitro antifungal activity of plant extracts obtained with alternative organic solvents against Rhizoctonia solani Kühn. Ind Crops Prod. 2010;32(3):324-8.

\section{Publisher's Note}

Springer Nature remains neutral with regard to jurisdictional claims in published maps and institutional affiliations.
Ready to submit your research? Choose BMC and benefit from:

- fast, convenient online submission

- thorough peer review by experienced researchers in your field

- rapid publication on acceptance

- support for research data, including large and complex data types

- gold Open Access which fosters wider collaboration and increased citations

- maximum visibility for your research: over $100 \mathrm{M}$ website views per year

At BMC, research is always in progress.

Learn more biomedcentral.com/submissions 